\title{
Unit Visualizations for Visual Storytelling
}

\author{
Antoine Béland \\ Le Devoir \\ abeland@ledevoir.com
}

\author{
Thomas Hurtut \\ Polytechnique Montréal \\ thomas.hurtut@polymtl.ca
}
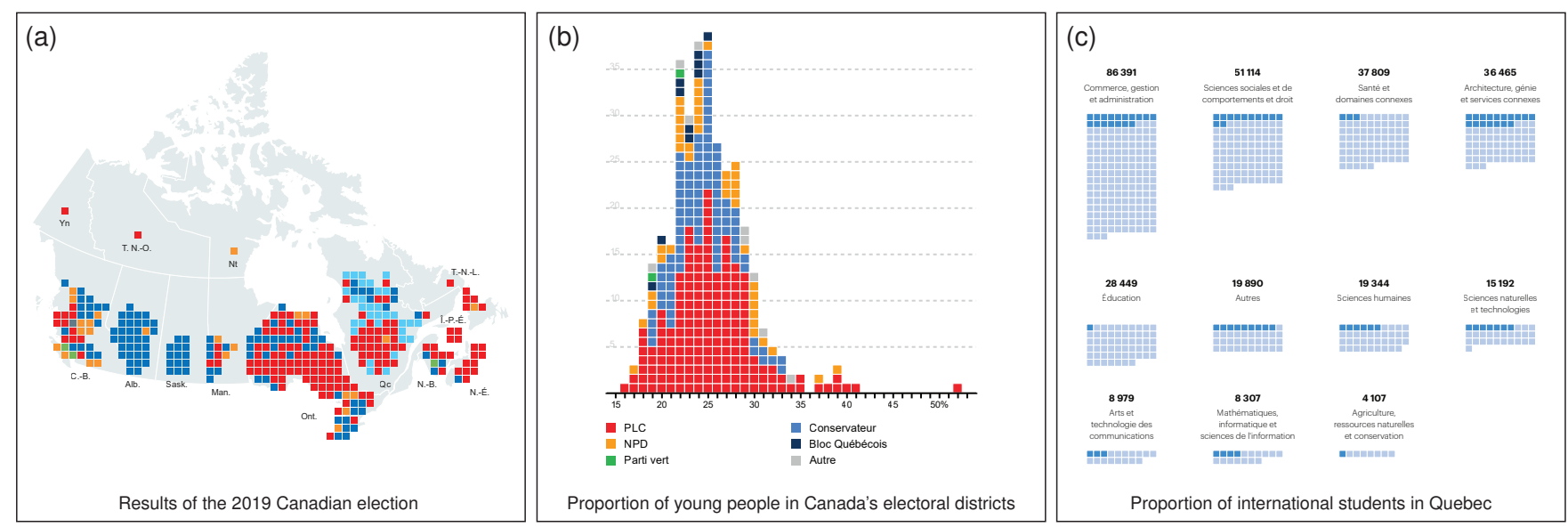

Figure 1: Three examples of unit visualization designs recently used in some Le Devoir's visual narratives.

\begin{abstract}
This case study is conducted by Le Devoir, a French-Canadian independent daily newspaper based in Montreal. This newspaper gathers about 50 journalists and attracts around 1 million readers every week. In the last six months, we published several visual stories using uniform unit visualizations. This type of visualization represents each data item as a specific visual element of constant size (e.g. square, circle, etc.). This case study details why unit visualizations can be particularly efficient representations for data-based visual stories. We also highlight the challenges and perspectives of such practice.
\end{abstract}

\section{KEYWORDS}

Unit visualization, journalism, storytelling.

\section{INTRODUCTION}

This case study focuses on three visual stories recently published in Le Devoir. Figure 1 illustrate some of the unit visualizations used in these stories.

The first story ${ }^{1}$ is based on a cartogram (Figure 1a) of the Canada's 338 federal electoral districts that shows the results of the 2019 Canadian election. Each square represents a

\footnotetext{
${ }^{1}$ https://www.ledevoir.com/documents/special/19-10_nouvelle-chambredes-communes-expliquee/index.html
}

specific district and the color used is associated with the winning political party of this district. The squares are located as near as possible of their real geographic locations.

The second story ${ }^{2}$ includes a distribution (Figure $1 \mathrm{~b}$ ) showing the proportion of young people (aged between 15 and 34 years old) for every Canada's federal electoral district. Each district is encoded as a square, where the color represents the winning political party for the 2015 Canadian election, and his position along the horizontal $x$-axis shows the percentage of young people living there.

The last story ${ }^{3}$ is based on a waffle chart layout (Figure 1c), showing the proportion of international students in Quebec compared to the Canadian students. Each square represents 500 students. Squares are grouped by study fields, and groups are sorted out by students counts. The square's color is used to distinguish the international students (dark blue) and the residents (light blue).

\section{WHY UNIT VISUALIZATIONS AND STORYTELLING FIT WELL?}

Three characteristics of unit visualizations make them efficient for visual narratives in mass media journalism: (1) they are intuitive; (2) they are adapted to storytelling and

\footnotetext{
${ }^{2}$ https://www.ledevoir.com/documents/special/19-08_jeunes_electeurs_ can/index.html

${ }^{3}$ https://www.ledevoir.com/documents/special/19-11_programmesetudiants-etrangers-quebec/index.html
} 
transitions due to their inherent flexible layouts; (3) they are adapted to visualization onboarding, due to their separable structure. We detail in this section how these aspects support the three stories of this case study.

Unit visualizations are often intuitive. Unit visualizations intuitively help data understanding because they reduce the abstraction between the visual marks and the data items (one-to-one correspondence) [4,5]. Each visual unit encodes a single reality (e.g. an electoral district Figure 1ab, a homogeneous group of students in Figure 1c). They also display good perceptual properties. Their atomicity facilitates the search of specific data items, either highlighted ones (foreign students in Figure 1c) or noticeably different results (orange district in Alberta Province, Western Canada, Figure 1a), and their tracking during transitions [3].

Unit visualizations' spatial mapping are inherently flexible. Because of their singularity and constant size, the spatial mapping of the visual marks can be easily changed without breaking apart the item correspondence. This helps the user to keep a narrative thread along the storytelling and avoids to lead him astray. A typical mapping transformation is the subdivision into groups according to common properties (e.g. foreign students vs. others in Figure 1c for each study field). While users cannot follow a very high number of visual items, the overall gist of uniform groups can still be perceived (such as in the possible transition from Figure 1a to Figure 1b). A popular interactive storytelling technique, extensively used online, is scrollytelling, where the content of the story appears or changes progressively through transitions as users scroll down [1]. This technique, combined to unit visualizations' flexibility, enhances the user engagement. It is based on a nowadays ubiquitous gestured interaction, and it gives a strong sense of control and fluidity over the narration flow, triggering an enjoyable discoverability perception. Besides, this format facilitates cross-platform stories (mobile, desktop, tablet) as a result of its general interaction. This is crucial in our context since around $60 \%$ of our online readers are on mobile devices.

Unit visualizations are easily separable. Visualization literacy cannot be assumed among mass media readers. Quickly and efficiently communicating how to read a given visualization is therefore a crucial preliminary step, otherwise the risk of misinterpretation is high [2]. A kind of visualization onboarding strategy should thus be thought of. In that context, storytelling has been identified as a promising onboarding method since it inherently embeds a building-up process allowing to progressively distill information [6]. We believe that this narrative essence combined to the easily separable nature of unit visualization foster the onboarding potential. By allowing to transmit incrementally both the mapping and the data items, the reader can be guided more safely throughout the understanding of the information that is being visually delivered. Transitions and annotations can also greatly support these learning phases. In Figure $1 \mathrm{~b}$ for instance, we first introduced the $x$-axis (percentage of young people) with two outliers (electoral districts having the minimal and maximum values) and no political party encoding. In a second narrative step, the Y-axis appears, revealing the sedimentation nature of the distribution. And in a final step, we introduced the color mapping which encodes political parties.

\section{CHALLENGES}

We went through several challenges when using unit visualizations for storytelling. First, unit visualizations can become visually cluttered if there are too much data items encoded at once. The broad usage of mobile devices increases this issue because of their low-resolution screens. To mitigate this problem, some data items must be aggregated into a larger group to reduce the number of visual elements. However, the aggregation should seek to facilitate the cognitive load for the reader, for instance by using a number of aggregated elements that can be easily understood and handled (e.g. 100, 500,1000 ). This strategy is used with the third story (Figure 1c), where 500 students are aggregated into one visual element.

Considering that each element should be visually encoded, the resulting visualization might not be scalable for all devices. The Scalable Vector Graphics (SVG) format is widely used to draw graphics on web browsers. However, this format is not adapted to draw thousand of items quickly, especially on mobile devices. In this case, another approach should be taken for the rendering, such as using a web canvas element.

A unit visualization is sometimes less adapted for a specific dataset or targeted message than a so-called aggregated visualization. We observed that unit visualizations are mostly useful when we want the readers to understand specific data item and decode its value (e.g. a unit, a person, a currency, a region, etc.). We experimented stories combining both types of visualizations with entangled transitions, for instance going from a unit visualization of electoral district to a histogram summing up the results aggregated for each political party. The representation's choice of each visualization and their succession through the narration are therefore important steps of the design process, depending on the targeted audience and communication goal.

Considering unit visualizations, some design guidelines and principles can be developed. For instance, in unit based cartograms (such as Figure 1a), the visual elements location should be close to the real position to mitigate the risk of possible misinterpretation induced by geographical distortions. Including familiar topographic cues such as province 
borders, or a natural asset (e.g. a river path) also help the reader decode the information. When the visual items must be grouped into different categories (e.g. Figure 1c), the resulting clusters should facilitate the data reading too. In this case, the clusters can use the same items count for the width, and an understandable and rememberable number for this items count (e.g. 5, 10, 20). Also, the clusters can be sorted by ascending or descending order to let easily catch the important elements.

\section{CONCLUSION AND PERSPECTIVES}

Although several issues need to be addressed when using unit visualizations for visual storytelling, we noticed that this narrative format provides multiple benefits to Le Devoir. The user engagement is usually higher than traditional articles. Feedback on social medias is also mostly positive. These stories were often praised as innovative visual essays for our newsroom. It thus provides a crucial distinguishing factor in our competitive industry. The flexibility of unit visualizations also allows a certain amount of project templating which enable us to produce these stories efficiently on a timely basis. Among the perspectives and possible future works, we believe more complex interactions could be explored to increase the engagement. Controlled user studies could also be done to qualify and quantify how unit visualizations are efficient in the context of storytelling with news readers, compared to possible equivalent representations using aggregated design. These studies should focus on the user's understanding, engagement and feedback.

\section{REFERENCES}

[1] Lorenzo Amabili. 2019. From Storytelling To Scrollytelling: A Short Introduction and Beyond*. https://medium.com/nightingale/fromstorytelling-to-scrollytelling-a-short-introduction-and-beyondfbda32066964

[2] Alberto Cairo. 2019. How charts lie. W. W. Norton \& Company.

[3] Pierre Dragicevic, Anastasia Bezerianos, Waqas Javed, Niklas Elmqvist, and Jean-Daniel Fekete. 2011. Temporal Distortion for Animated Transitions. In Proc. SIGCHI Conference on Human Factors in Computing Systems (CHI '11). 2009-2018.

[4] Steven Drucker and Roland Fernandez. 2015. A Unifying Framework for Animated and Interactive Unit Visualizations. Technical Report. Microsoft Research. https:/www.microsoft.com/enus/research/publication/a-unifying-framework-for-animated-andinteractive-unit-visualizations/

[5] Deokgun Park, Steven M Drucker, Roland Fernandez, and Niklas Elmqvist. 2018. Atom: A grammar for unit visualizations. IEEE Transactions on Visualization and Computer Graphics 24, 12 (2018), 3032-3043.

[6] Christina Stoiber, Florian Grassinger, Margit Pohl, Holger Stitz, Marc Streit, and Wolfgang Aigner. 2019. Visualization Onboarding: Learning How to Read and Use Visualizations. In IEEE Workshop on Visualization for Communication. 